\title{
Optimal Allocation of Bandwidth for Minimum Battery Consumption
}

\author{
Qinghua Zhao, Pamela C. Cosman, and Laurence B. Milstein \\ Department of Electrical and Computer Engineering, University of California, San Diego. 9500 Gilman Drive, \\ La Jolla, CA 92093-0407; \{qizhao,pcosman,lmilstein\}@ucsd.edu
}

\begin{abstract}
In general, a power amplifier utilizes battery energy more efficiently with a higher transmission power. For a given message, a given bandwidth constraint and a given performance constraint, different allocations of the bandwidth among source coding, channel coding and modulation result in different amounts of battery usage. We propose a method to optimize the bandwidth allocation and minimize the battery consumption due to transmission. Our results show that with the optimal allocation, significant reduction in battery consumption can be achieved without sacrificing the system performance.
\end{abstract}

Keywords - Bandwidth allocation, battery consumption optimization, coded modulation, transmission over wireless channels.

\section{INTRODUCTION}

$\mathrm{B}$ ATTERY usage for portable devices in mobile communications has been studied from various aspects. For example, [1] proposed to exploit the networking infrastructure, by shifting part of the computation off the mobile unit, thus conserving the battery consumption of the mobile. In [2], a battery conservation management method is proposed to minimize the battery consumption by switching the battery to different operational modes depending on the network packet transmission properties. These two examples minimize the battery consumption from an algorithmic and protocol point of view. Battery consumption optimization utilizing properties of the hardware are also proposed as in [3], where the CPU of the mobile is scheduled to run at different frequencies according to the load; and also as in [4], where the charge recovery properties of the battery under bursty discharge conditions are exploited. In this paper, we exploit a characteristic of the power amplifier of the mobile units. By optimally allocating the bandwidth to source coding, channel coding and the modulation constellation, the power amplifier can work more efficiently and thus reduce the battery consumption of the mobile unit.

\section{SYSTEM OVERVIEW}

The system block diagram is given in Figure 1. The system consists of a uniform source (a scalar source uniformly distributed on $[0,1)$ ), a source coder, a channel coder, and a modulator.

There are $W$ source symbols that need to be transmitted. The source encoder codes each of the $W$ symbols into $r_{s}$ bits, where $r_{s}$, in bits-per-symbol, is the source code rate. These $W r_{s}$ bits are coded to $\frac{W r_{s}}{r_{c}}$ bits by the channel coder with channel code

Acknowledgment: This research was partially sponsored by the Center for Wireless Communications of UCSD, the UC Discovery program of the State of California, and Ericsson Wireless Communications Incorporated.

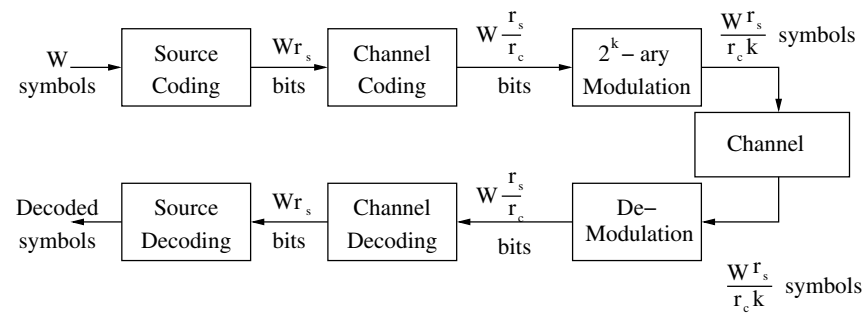

Fig. 1. System overview.

rate $r_{c}$, and then mapped to $\frac{W r_{s}}{r_{c} k}$ symbols to be transmitted across the channel with a $2^{k}$-ary modulation scheme, where $k$ is the alphabet size. At the receiver, noise corrupted symbols are recovered and then decoded to the best source code points. The performance of the system is measured by the endto-end distortion, $D$, between the transmitted symbols and the decoded symbols.

There are two constraints on the system. First, the bandwidth is limited to $\bar{C}_{0} H z$, which translates to a transmission rate that is upper bounded by $C$ symbols per second after pulse shaping. Second, the system has to achieve an end-to-end distortion $D \leq$ $D_{0}$. We would like to find the optimal source code rate, $\hat{r}_{s}$, the optimal channel code rate, $\hat{r}_{c}$, and the optimal alphabet size, $\hat{M}=2^{\hat{k}}$, that minimize the battery drain, $B_{d}$, in coulombs.

\section{ANALYSIS}

\section{A. System Performance}

We assume that the source symbols are scalars uniformly distributed over $[0,1)$, and that the source encoder is an $r_{s}$-bit uniform quantizer concatenated with a random index assignment. From [5], we know that the system performance, i.e., end-toend distortion, is

$$
D\left(r_{s}, r_{c}, k, P_{\text {out }}\right) \approx \frac{1}{12} 2^{-2 r_{s}}+\frac{1}{6} P_{e}\left(r_{s}, r_{c}, k, P_{\text {out }}\right),
$$

where $P_{\text {out }}$ is the output power of the amplifier, i.e., the power of the transmitted symbol, and $P_{e}$ is the index error rate. In (1), note that here we have explicitly expressed the probability of error as a function of $P_{\text {out }}$.

\section{B. Battery Drain}

We make the following assumptions:

- The battery drain primarily comes from the power amplifier of the transmitter, meaning the computation power consumption from the system is negligible. 
- The power amplifier is operating in its linear region.

- The battery is ideal, i.e., it has a constant voltage and a constant capacity, where neither of them depend on the value of the load [6]. Here the battery capacity refers to the amount of energy a battery can deliver under normal operating conditions, usually expressed in ampere-hours, watt-hours, or similar units. With these assumptions, the battery drain is given by

$$
B_{d}=\int_{0}^{T_{x}} I_{\text {Batt }}\left(P_{\text {out }}(t)\right) d t, \quad P_{\text {out }} \in\left[0, P_{\text {sat }}\right]
$$

where $P_{\text {out }}$ is the instantaneous output power of the amplifier, $P_{\text {sat }}$ is the saturation power of the amplifier, $I_{B a t t}$ is the instantaneous battery discharge current, and

$$
T_{x}=\frac{W r_{s}}{r_{c} k C}
$$

is the total transmission time.

The properties of the power amplifier play a crucial role in formulating this optimization problem. Reference [7, Fig.2] gives the battery discharge current, $I_{B a t t}$, as a function of the output power of the amplifier, $P_{\text {out }}$ (in $\mathrm{dBm}$ ), for a typical cellular band system. The figure given in [7], as well as other similar amplifier figures in the literature, can be modeled by

$$
I_{\text {Batt }}\left(P_{\text {out }}\right) \approx f_{0}+f_{1} e^{f_{2} P_{\text {out }, d B}}=f_{0}+f_{1} e^{10 f_{2} \cdot \log _{10} P_{\text {out }}} .
$$

Equivalently,

$$
I_{\text {Batt }}\left(P_{\text {out }}\right) \approx f_{0}+f_{1} P_{\text {out }}^{f_{3}} .
$$

The coefficients $f_{i}, i=0,1,2,3$, can be determined for specific amplifiers, and $f_{3}=\frac{10 f_{2}}{\ln 10}$. For example, in [8], we modeled the measured results in [7, Fig. 2] as

$$
I\left(P_{\text {out }}\right)=f_{0}+f_{1} e^{0.1 P_{\text {out }, d B}},
$$

where $f_{0}=0.0321, f_{1}=0.638$. Both the original measured data and the fitted curve are plotted in Figure 2. It is worth noting that in the area of amplifier design, a good amplifier should be as linear as possible, corresponding to a smaller $f_{2}$.

The output power region shown in Figure 2 gives the normal output power range of a wireless unit [7]. From this curve, we can see that for every $3 d B$ increase, or doubling, of the output power $P_{\text {out }}$, the battery current $I_{b a t t}$ increases less than a factor of two. In other words, with this amplifier, a higher transmission power utilizes the battery more efficiently. This is true in general for amplifiers. More explicitly, if $P_{1}>P_{2}$ and $P_{1} \cdot T_{1}=P_{2} \cdot T_{2}$, transmitting with power $P_{1}$ for $T_{1}$ seconds uses less charge from the battery than transmitting with power $P_{2}$ for $T_{2}$ seconds. This property of the power amplifier directly affects the optimization results. For example, with a higher alphabet size, there are fewer symbols to transmit for the given message, thus each symbol can be transmitted with a higher power for the same transmission energy, and consequently uses the amplifier more efficiently. On the other hand,

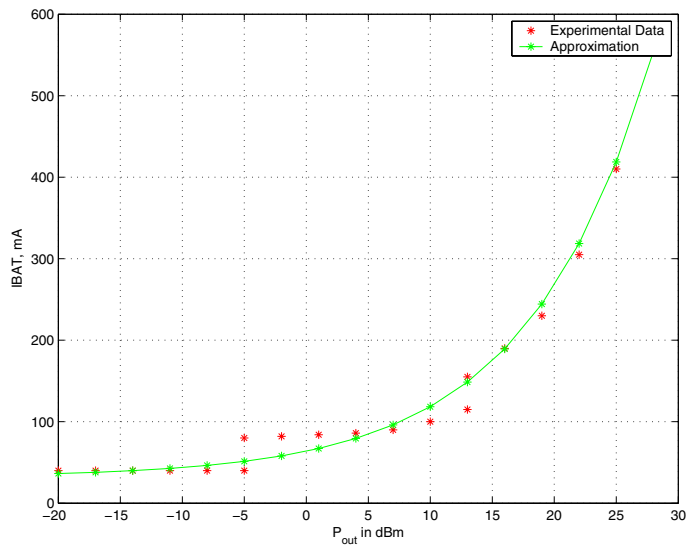

Fig. 2. Measured and modeled amplifier battery current property of [7, Fig.2].

a higher order two-dimensional constellation results in a larger probability of error for a given $E_{b} / N_{o}$, which counterbalances the advantage given by the amplifier. Thus, there exists an optimal $k$ for the system.

The battery consumption is given by

$$
\begin{aligned}
B_{d} & =\int_{0}^{T_{x}} I_{\text {Batt }}\left(P_{\text {out }}(t)\right) d t \\
& =\sum_{i=0}^{\frac{T_{x}}{T_{s}}-1} \int_{0}^{T_{s}} I_{\text {Batt }}\left(P_{\text {out }}\left(t+i T_{s}\right)\right) d t \\
& =T_{x} \cdot \frac{T_{s}}{T_{x}} \sum_{i=0}^{\frac{T_{x}}{T_{s}}-1} I_{\text {Batt }, \text { avg }}\left(P_{\text {out }}\left(t+i T_{s}\right)\right) \triangleq T_{x} \cdot \bar{I}_{\text {Batt }},
\end{aligned}
$$

where $\bar{I}_{B a t t}$ is the equivalent constant battery current, and $I_{B a t t, a v g}$ is the battery current averaged over the $i$-th symbol duration, defined as

$$
I_{\text {Batt }, \text { avg }}(i) \triangleq \frac{1}{T_{s}} \int_{0}^{T_{s}} I_{\text {Batt }}\left(P_{\text {out }}\left(t+i T_{s}\right)\right) d t .
$$

Assume a) most of the energy of the shaping pulse resides in the time duration $\left[0, T_{s}\right]$, so that the energy of the shaping pulse outside of the symbol duration can be ignored, and, b) there is no filtering in the amplifier, so the transition between the symbols does not cause significant distortion to the shape of the pulse in each symbol duration. Then we have

$$
P_{\text {out }}\left(t+i T_{s}\right)=P_{i} \cdot h(t),
$$

where $P_{i}$ is the average power of the $i$ th transmitted symbol, and $h(t)$ is the shaping pulse which has energy 1 over $\left[0, T_{s}\right]$. Substituting (9) into (8), we have, using (4),

$I_{\text {Batt }, \text { avg }}(i)=\frac{1}{T_{s}} \int_{0}^{T_{s}}\left(f_{0}+f_{1} e^{10 f_{2} \cdot \log _{10}\left(P_{i} h(t)\right)}\right) d t$ 


$$
\begin{aligned}
& =f_{0}+f_{1} e^{10 f_{2} \cdot \log _{10} P_{i}} \cdot \frac{1}{T_{s}} \int_{0}^{T_{s}} e^{10 f_{2} \cdot \log _{10} h(t)} d t \\
& =f_{0}+f_{1} h_{0} e^{10 f_{2} \cdot \log _{10} P_{i}},
\end{aligned}
$$

where

$$
h_{0} \triangleq \frac{1}{T_{s}} \int_{0}^{T_{s}} e^{10 f_{2} \cdot \log _{10} h(t)} d t
$$

is a constant determined by the shaping pulse, $h(t)$.

With the assumptions that the symbols in the constellation are equally probable, and that the number of symbols transmitted, $T_{x} / T_{s}$, is much larger than the constellation size $2^{k}$, we have

$$
\bar{I}_{\text {Batt }} \triangleq \frac{T_{s}}{T_{x}} \sum_{i=0}^{\frac{T_{x}}{T_{s}}-1} I_{\text {Batt }, a v g}(i) \approx \frac{1}{2^{k}} \sum_{j=0}^{2^{k}-1} I_{\text {Batt }, \text { avg }}^{(j)},
$$

where $I_{\text {Batt,avg }}^{(j)}$ is the average battery current in the interval $\left[0, T_{s}\right]$, assuming the $j$ th point in the constellation is transmitted during that interval. Let $\bar{P}$ be the average power of the constellation, and assume the $j$ th symbol of the constellation has power $P^{(j)}=a_{j} \bar{P}$. Then, from (12),

$$
\begin{aligned}
\bar{I}_{\text {Batt }} & \approx \frac{1}{2^{k}} \sum_{j=1}^{2^{k}-1} \bar{I}_{\text {Batt }, a v g}^{(j)} \\
& =\frac{1}{2^{k}} \sum_{j=0}^{2^{k}-1}\left(f_{0}+f_{1} h_{0} e^{10 f_{2} \cdot \log _{10} P^{(j)}}\right) \\
& =f_{0}+f_{1} h_{0} M_{0} e^{10 f_{2} \cdot \log _{10} \bar{P}}
\end{aligned}
$$

where

$$
M_{0} \triangleq \frac{1}{2^{k}} \sum_{j=0}^{2^{k}-1} e^{10 f_{2} \cdot \log _{10} a_{j}} .
$$

Together with Equation (7), we have

$$
B_{d}=\frac{W r_{s}}{r_{c} k C} \cdot\left(f_{0}+f_{1} h_{0} M_{0} e^{10 f_{2} \cdot \log _{10} \bar{P}}\right)
$$

or

$$
B_{d}=\frac{W r_{s}}{r_{c} k C} \cdot\left(f_{0}+f_{3} h_{0} M_{0} \bar{P}^{f_{3}}\right) .
$$

Note that $M_{0}$ is determined by the constellation. For example, for all PSK modulations, $a_{j}=1$, and therefore $M_{0}=1$. The values of $M_{0}$ for some QAM constellations for the model in Figure 2, where $f_{2}=0.1$, are listed in Table I. We call $M_{0}$ the constellation factor. For the same average power, a constellation with a smaller $M_{0}$ induces a smaller battery drain. For example, for the same transmission power, 64QAM will induce a smaller battery drain than 32QAM, 16QAM, and QPSK. In general, the higher the order of the constellation, the smaller is $M_{0}$, i.e., the more efficiently the battery works. On the other hand, from the performance point of view, for the same average power, the higher the order of the constellation, the smaller

\begin{tabular}{c|ccccc}
\hline & & & & & \\
& QPSK & 8QAM & 16QAM & 32QAM & 64QAM \\
\hline$M_{0}$ & 1 & 0.9345 & 0.9470 & 0.9454 & 0.9387 \\
\hline
\end{tabular}

TABLE I

SOME $M_{0}$ VALUES FOR QAM MODULATIONS.

is the Euclidean distance between different symbols, and the larger is the error rate. To meet the system performance requirement, a higher order constellation requires a larger average transmission power.

In summary, (15) or (16) gives the battery consumption for a given constellation and a given shaping pulse, subject to the following assumptions:

- The symbols in the constellation are equally probable.

- The number of transmitted symbols is much larger than the constellation size.

- The energy of the shaping pulse in the intervals $(-\infty, 0)$ and $\left(T_{s},+\infty\right)$ can be ignored.

- The distortion of the signal at the signal boundaries, i.e., at $i T_{s}$, where $i$ is an integer, is negligible.

\section{Optimization}

Our goal is to optimize Equation (15), subject to the constraint given by (1). The bandwidth constraint is embedded in the transmission time $T_{x}$, as shown in Equation (3), and thus is in Equation (15).

In [9], we show that for most practical systems, the source code rate that minimizes the battery consumption is either $\hat{r}_{s}=$ $r_{0}$ or $\hat{r}_{s}=r_{0}+1$, where $r_{0}$ is the smallest integer that satisfies $\frac{1}{12} 2^{-2 r}<D_{0}$. With the optimal source code rate determined, we only need to find the optimal channel code and constellation size that produce the smallest $B_{d}$ and satisfy

$$
\begin{aligned}
& D_{0} \geq \frac{1}{12} 2^{-2 \hat{r}_{s}}+\frac{1}{6} P_{e}\left(\hat{r}_{s}, r_{c}, k, P_{\text {out }}\right) \\
\Longrightarrow & D_{0}-\frac{1}{12} 2^{-2 \hat{r}_{s}} \geq \frac{1}{6} P_{e}\left(\hat{r}_{s}, r_{c}, k, P_{\text {out }}\right) \\
\Longrightarrow & \bar{D}_{0} \triangleq 6\left(D_{0}-\frac{1}{12} 2^{-2 \hat{r}_{s}}\right) \geq P_{e}\left(\hat{r}_{s}, r_{c}, k, P_{\text {out }}\right) .
\end{aligned}
$$

For any given 2-tuple $\left(r_{c}, k\right)$, since $P_{e}$ is a continuous decreasing function of $P_{\text {out }}$, the smallest transmission power that satisfies (17) satisfies it with equality. Also, since the battery consumption in (15) is an increasing function of $P_{\text {out }}$, the smallest $P_{\text {out }}$ that satisfies (17) also gives the least battery consumption for a given $\left(r_{c}, k\right)$. Therefore, the inequality performance constraint is equivalent to the equality constraint given by

$$
\bar{D}_{0}=P_{e}\left(r_{s}, r_{c}, k, P_{\text {out }}\right) \text {. }
$$

The error performance of the concatenated convolutional code and the modulation is not a simple function of either the channel code rate, $r_{c}$, or the modulation alphabet size, $k$. Also, the structure of the convolutional code and the mapping of the channel code bits to the modulation symbols are significant factors that determine the performance. These properties make it 
hard to obtain closed-form expressions for the error probability. In this section, we present an optimization procedure for arbitrary codes without going into the details of either the convolutional code structure or the mapping of the modulated signals. In the next section, we illustrate the optimization results for some specific systems.

Aside from the constellation factor, $M_{o}$, defined in the previous section, there are two other properties of a code that are of importance to the optimization. The first one is the number of source bits per modulated symbol, $\alpha \triangleq r_{c} k$, which directly determines the message duration $T_{x}$ when both $W$ and $r_{s}$ are known. The larger $\alpha$ is, the shorter is the time needed to transmit the given message of length $W$.

In [10], the effective coding gain is used to measure the reduction of $E_{b} / N_{o}$ (or $S N R$ ) of coded modulation schemes for a given target error performance. It is used to compare the performance of different codes with the same information rate in [10]. In this paper, we define a similar concept, SNR coding gain, $\Gamma$, to compare the $S N R$ requirement to achieve a target error performance. The difference between $\Gamma$ and the effective coding gain defined in [10] is that $\Gamma$ allows the comparison between codes with different information rates. This way, we can compare the energy usage among codes with these different rates. To elaborate, for a given error performance, if code 2 has a $\Gamma d B$ gain over code 1 , then the signal-to-noise ratios of these two codes that are needed to achieve this performance have the following relationship:

$$
S N R_{1, d B}-S N R_{2, d B}=\Gamma .
$$

For a given noise figure of the receiver and a given transmission symbol rate, $S N R \propto \frac{E_{s}}{N_{o}} \propto \bar{P} T_{s}$, where $E_{s}$ is the average signal energy, $N_{o} / 2$ is the two-sided noise power density, $\bar{P}$ is the average transmission power, and $T_{s}$ is the symbol duration. Note that

$$
\begin{aligned}
\bar{P}_{1, d B}-\bar{P}_{2, d B} & =\left(\frac{E_{s}}{N_{o}}\right)_{1, d B}-\left(\frac{E_{s}}{N_{o}}\right)_{2, d B} \\
& =S N R_{1, d B}-S N R_{2, d B}=\Gamma .
\end{aligned}
$$

It is easy to see that the SNR coding gain, $\Gamma$, indicates a level of power reduction. Or, more specifically, for a given error performance, if system 1 has an average power $\bar{P}_{1}$, and system 2 has $\Gamma d B$ SNR gain over system 1, then system 2 requires an average transmission power of

$$
\bar{P}_{2}=\bar{P}_{1} \times 10^{-0.1 \Gamma},
$$

to achieve the same error performance.

Our general optimization problem is formulated as follows: Assume system 1, with channel code rate $r_{c}$ and alphabet size $k$, satisfies the performance constraint given in (18). Its battery consumption is given by (15) with $P_{\text {out }}=\bar{P}$. If we change the channel code rate to $r_{c}^{\prime}$ and the alphabet size to $k^{\prime}$, what is the gain, $\Gamma$, of code 2 over code 1 , that is needed in order for system 2 to consume at most the same battery charge as system 1 ?
Assume the constellation factors of code 1 and code 2 are $M_{0}$ and $M_{0}^{\prime}$, respectively. Also, assume $\bar{P}_{d B}-\bar{P}_{d B}^{\prime}=\Gamma$, $\alpha=r_{c} k$ and $\alpha^{\prime}=r_{c}^{\prime} k^{\prime}$. From (16), we want

$$
\begin{aligned}
& \left.\frac{W r_{s}}{r_{c}^{\prime} k^{\prime} C} \cdot\left(f_{0}+f_{1} h_{0} M_{0}^{\prime} \bar{P}^{\prime} f_{3}\right) \leq \frac{W r_{s}}{r_{c} k C} \cdot\left(f_{0}+f_{1} h_{0} M_{0} \bar{P}^{f_{3}}\right)\right) \\
& \Longleftrightarrow \bar{P}^{\prime f_{3}} \leq\left(\left(\frac{\alpha^{\prime}}{\alpha}-1\right) \frac{f_{0}}{f_{1} h_{0} M_{0}}+\frac{\alpha^{\prime}}{\alpha} \bar{P}^{f_{3}}\right) \frac{M_{0}}{M_{0}^{\prime}} \\
& \Longleftrightarrow 10 \log _{10} \bar{P}-10 \log _{10} \bar{P}^{\prime} \geq-\frac{10}{f_{3}} \log _{10} \frac{M_{0}}{M_{0}^{\prime}}+10 \log _{10} \bar{P} \\
& -\frac{10}{f_{3}} \log _{10}\left(\left(\frac{\alpha^{\prime}}{\alpha}-1\right) \frac{f_{0}}{f_{1} h_{0} M_{0}}+\frac{\alpha^{\prime}}{\alpha} \bar{P}^{f_{3}}\right) \\
& \Longleftrightarrow \Gamma=P_{d B}-P_{d B}^{\prime} \geq-\frac{10}{f_{3}} \log _{10} \frac{M_{0}}{M_{0}^{\prime}} \\
& +\frac{10}{f_{3}} \log _{10}\left(\frac{\bar{P}^{f_{3}}}{\left(\frac{\alpha^{\prime}}{\alpha}-1\right) \frac{f_{0}}{f_{1} h_{0} M_{0}}+\frac{\alpha^{\prime}}{\alpha} \bar{P}^{f_{3}}}\right)
\end{aligned}
$$

The right hand side of (22) shows how much gain the second system has to have in order to result in at most the same battery drain as the first system. If the gain is larger than $\Gamma$ as given in (22), the second system consumes less battery charge, and vice versa. This equation can be used to find the optimal system among a set of possible codes, as long as we know the parameters $M_{0}, \alpha$ and $\Gamma$.

\section{RESUlts}

In this section, we present examples of the optimization results for the trellis codes given in [11, Table III]. There, for each binary convolutional code, Ungerboeck searched different mappings from the convolutionally coded bitstream to PSK, AMPM, and QAM signal constellations for the largest minimum distance. Simulation results of the error performance of these "good" codes are also given. For each row in [11, Table III], the convolutional codes are the same for different modulations, so the number of states for each trellis is the same, which in turn provides the same channel decoding complexity for a fair comparison across different trellis codes. Figure 3 shows the constellations that are used in [11], and are subsequently used in this section. The 8QAM and 32QAM constellations in Table I are also shown here. Note that the 8AMPM and 32AMPM constellations used in this section are different from the 8QAM and the cross 32QAM constellations shown in Figure 3.

For fixed $W$ and $r_{s}$, Table II shows the tradeoffs between different trellis codes that are given on the second line of [11, Table III]. In Table II, all transmission times are normalized to the transmission time of the uncoded BPSK constellation, $T_{0}=W r_{s}, \bar{I}_{\text {Batt }}$ is calculated from $\bar{P}_{\text {out }}$ by using Equation (13), where we assume $h_{0}$ from Equation (11) equals 0.9 , and $\Gamma$ is the SNR gain compared to the coded 8AMPM constellation, whose average transmission power is assumed to be 6 $d B m$. The procedure for calculating $\Gamma$ for each code is shown in Table III, where signal-to-noise ratios at a bit error rate of $10^{-5}$ are used. Since the error rate curves for all constellations 


\begin{tabular}{|c|c|c|c|c|c|c|c|c|c|}
\hline Modulation & $r_{c}$ & $k$ & $M_{0}$ & $\alpha$ & $T_{x}$ & $\begin{array}{c}\Gamma \\
d B\end{array}$ & $\begin{array}{l}\bar{P}_{\text {out }} \\
d B m\end{array}$ & $\begin{array}{c}\bar{I}_{\text {Batt }} \\
m A\end{array}$ & $\begin{array}{l}B_{d}=T_{x} \bar{I}_{\text {Batt }} \\
\text { millicoulomb }\end{array}$ \\
\hline 8AMPM & $2 / 3$ & 3 & 0.9470 & 2 & $T_{0} / 2$ & 0.0 & 6 & 81.4 & $40.7 T_{0}$ \\
\hline 16QAM & $3 / 4$ & 4 & 0.9470 & 3 & $T_{0} / 3$ & -2.9 & 8.9 & 98.0 & $32.7 T_{0}$ \\
\hline 32AMPM & $4 / 5$ & 5 & 0.9387 & 4 & $T_{0} / 4$ & -6.7 & 12.7 & 127.7 & $31.9 T_{0}$ \\
\hline 64QAM & $5 / 6$ & 6 & 0.9387 & 5 & $T_{0} / 5$ & -9.7 & 15.7 & 161.1 & $\overline{32.2 T_{0}}$ \\
\hline
\end{tabular}

TABLE II

BATTERy CONSUMPTION COMPARISON FOR TRELLIS CODES IN [11, TABLE III]. AMPLIFIER PROFILE FROM FigURE 2.

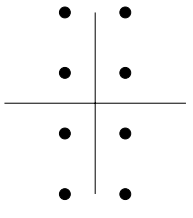

8-QAM

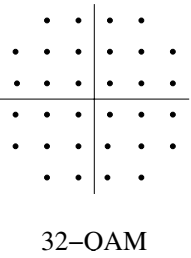

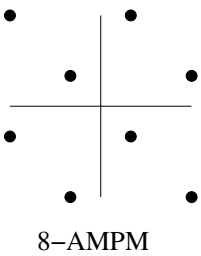

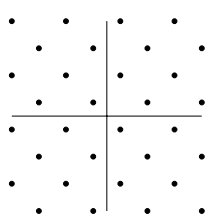

Fig. 3. Signal constellations

are virtually parallel for low bit error rate, these $\Gamma$ values for a different bit error probability in the parallel region will remain unchanged. All values in the table are in $d B$.

The data in the first row are obtained from [11], [12], [13],

\begin{tabular}{l|cccc}
\hline $\mathbf{a}:$ coded $2^{k}$-ary & 8AMPM & 16QAM & 32AMPM & 64QAM \\
\hline $\mathbf{b : ~ u n c o d e d ~} 2^{k-1}$-ary & QPSK & 8AMPM & 16QAM & 32AMPM \\
\hline$\left(\frac{E_{s}}{N_{o}}\right)_{\mathbf{a}}$ & 12.6 & 16.5 & 20.1 & 23.3 \\
$\left(\frac{E_{s}}{N_{o}}\right)^{\mathbf{a}}-\left(\frac{E_{s}}{N_{o}}\right)_{\mathbf{b}}$ & -3.0 & -4.0 & -3.8 & -4.0 \\
$\left(\frac{E_{s}}{N_{o}}\right)_{\mathbf{b}}$ & 9.6 & 12.5 & 16.3 & 19.3 \\
\hline & 0.0 & -2.9 & -6.7 & -9.7 \\
\hline
\end{tabular}

TABLE III

$\Gamma$ VALUES FOR DIFFERENT CODES COMPARED WITH CODED 8AMPM MODULATION.

[14]. Note that the value of $\left(\frac{E_{s}}{N_{o}}\right)_{\text {uncoded }}$ for 32AMPM obtained from [11] is an approximation.

From Table II, 32AMPM with a channel code rate of $4 / 5$ is the best trellis code among the four codes shown. We see that a higher order constellation needs to transmit the symbols with a higher power. But, since a) from the amplifier profile, the amplifier is more efficient for higher transmission power (e.g., $\bar{I}_{\text {Batt }}$ only increases by a factor of two when the transmission power increases by $9.7 \mathrm{~dB}$ in going from 8AMPM to 64QAM), and, b) the transmission time $T_{x}$ for the higher order constellation is shorter, there exists an optimal code which results in the least amount of battery consumption. The final optimization result in this table is the combination of the effects of a) and b).

Figure 4 shows the battery consumption for several different constellations. Each of the five curves corresponds to a different value of the average transmission power, $\bar{P}_{\text {out,coded } 8 \text { AMPM. }}$. For a given number of symbols per second, $C$, a given noise spectral density, $N_{o}$, and a given channel code as well as the bits-to-symbols mapping, specifying $\bar{P}_{\text {out,coded 8AMPM }}$ is equivalent to specifying the error performance constraint, $\bar{D}_{0}$, since they have a one-to-one correspondence. Here, we used $\bar{P}_{\text {out,coded 8AMPM }}$ instead of $\bar{D}_{0}$ to parameterize each curve. This is because for different values of $N_{o}$, the same $\bar{P}_{\text {out,coded 8AMPM }}$ corresponds to different values of $D_{0}$, but as long as $\bar{P}_{\text {out,coded } 8 \text { AMPM }}$ and the SNR coding gains between these constellations are the same, the corresponding curve in Figure 4 will be exactly the same.

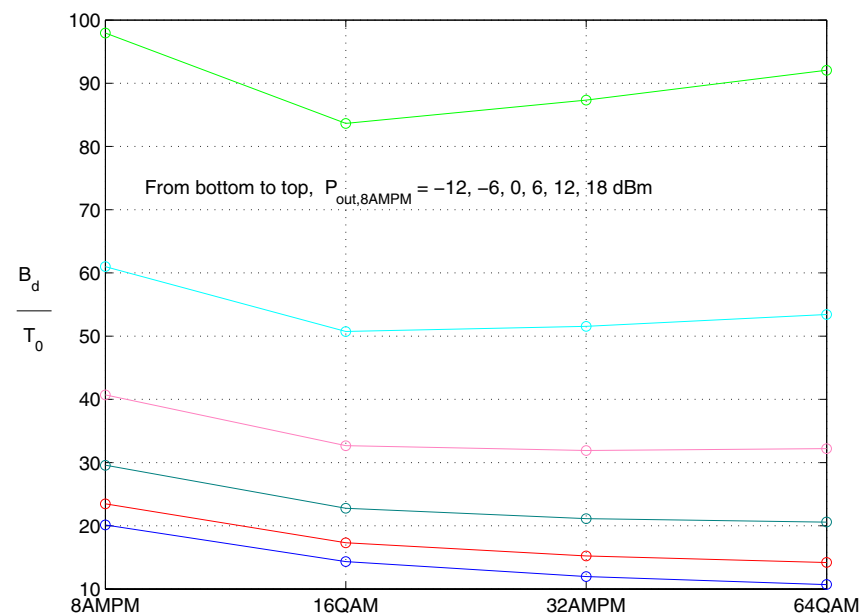

Fig. 4. Battery consumption for different $\bar{P}_{\text {out,coded 8AMPM }}$ for trellis codes in [11, Table III]. Amplifier profile from Figure 2.

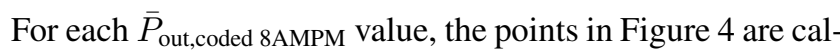
culated the same way as in Table III. The third curve from the bottom on Figure 4 shows the results from Table II. As in Table II, the battery drain is in millicoulombs, and is normalized by a factor of $\frac{1}{T_{0}}=\frac{1}{W r_{s}}$. As shown in Table II, the transmission times for the different codes are $T_{0} / 2, T_{0} / 3, T_{0} / 4$, and 
$T_{0} / 5$ for the constellations from left to right on the $\mathrm{x}$-axis. To be more explicit, take the bottom curve as an example. The bottom curve corresponds to a $\bar{P}_{\text {out,coded 8AMPM }}=-12 \mathrm{dBm}$. From Table II, we know the average powers of the other three constellations are $-12+\{2.9,6.7,9.7\}=\{-9.1,-5.3,-2.3\}$ $d B m$. Substituting these values into Equation 13, we obtain the $\bar{I}_{B a t t}$ values for each constellation. By multiplying these $\bar{I}_{B a t t}$ values with their individual transmission times, we arrive at the $B_{d}$ values shown in the plot.

For the bottom three curves in Figure 4, where the average transmission powers for all constellations are low compared with the other curves, the power amplifier is much more efficient for higher order constellations than for lower order constellations. The combined effect of the shorter transmission time and the more efficient power amplifier usage results in the 64QAM draining the least amount of battery charge among all four trellis codes.

As we move up to the fourth curve from the bottom, the transmission power increases for all constellations, and the amplifier becomes more efficient for all constellations, causing the advantage of the amplifier efficiency for higher order constellations to diminish. Even though the shorter transmission time advantage remains the same, the coded 64QAM is no longer the optimal code when $\bar{P}_{\text {out,coded } 8 \text { AMPM }}$ increases to $6 \mathrm{dBm}$. When $\bar{P}_{\text {out,coded 8AMPM }}$ increases to $12 \mathrm{dBm}$ (the fifth curve from the bottom), the optimal code among the four is coded 16QAM, with a channel code rate $r_{c}=3 / 4$. In both Table II and Figure 4, the amplifier profile in Equation (4), with $f_{0}=0.0321$, $f_{1}=0.038$, and $f_{2}=0.10$, is used.

From Figure 4, we can see that as transmission power increases in going from the bottom curve to the top curve, the advantage of the larger transmission power of the higher order constellation diminishes. But the advantage for the second amplifier profile diminishes slower than that of the first amplifier profile. Therefore, the second amplifier favors a system with larger constellations more than the first amplifier does.

In this paper, the examples are given only for the trellis codes studied in [11]. However, the optimization can be carried out for any codes as long as we know the constellation factor, $M_{0}$, the number of source bits-per-symbol, $\alpha=r_{c} k$, and the performance gain, $\Gamma$.

\section{CONCLUSIONS}

For the system described in this paper with a given bandwidth constraint and an end-to-end distortion constraint, we can find the source code rate, the channel code (among a given set of codes) and the modulation alphabet size which results in the least amount of battery drain in order to transmit a given source.

In most practical systems, the optimal source code rate, $\hat{r}_{s}$ will be either $r_{0}$ or $r_{0}+1$, where $r_{0}$ is defined as the smallest integer that satisfies $\frac{1}{12} 2^{-2 r}<D_{0}$ as proved in [9].

With the optimal source code rate $\hat{r}_{s}$, the concatenation of the convolutional code and the modulation can be characterized by three parameters: the constellation factor, $M_{0}$, the number of source coded bits-per-modulated symbol, $\alpha$, and the SNR coding gain, $\Gamma$. With these three parameters, we can find the optimal code among a set of given codes, and therefore the optimal channel code rate $\hat{r}_{c}$ and alphabet size $\hat{k}$ among the set.

When the transmission powers of all systems are small, the coded modulation with a larger constellation size has a significant amplifier efficiency advantage over a coded modulation with a smaller constellation size. In these cases, a coded modulation with a large constellation, which has a shorter transmission time, tends to consume less battery charge than a coded modulation with a small constellation size. As the transmission powers of all coded modulation schemes increase, the systems with smaller constellations become more efficient with respect to battery usage. In these cases, a smaller constellation, which has a lower average transmission power, tends to win over a larger constellation.

\section{REFERENCES}

[1] C. W. Probst, A. G. Gal, and M. Franz, "Code generating routers: a network-centric approach to mobile code," Proceedings of the IEEE 18th Annual Workshop on Computer Communications, pp. 179-186, oct. 2003.

[2] C. F. Chiasserini and R. R. Rao, "Improving energy saving in wireless systems by using dynamic power management," IEEE Transactions on Wireless Communications, vol. 2, pp. 1090-1100, sept. 2003.

[3] Y. Nakamoto, Y. Tsujino, and N. Tokura, "Adaptive battery conservation management using packet qos classifications for multimedia mobile packet communications," Proceedings of the IEEE Vehicular Technology Conference, vol. 2, pp. 834-838, sept. 2002.

[4] C. F. Chiasserini and R. R. Rao, "Improving battery performance by using traffic shaping techniques," IEEE Journal on Selected Areas in Communications, vol. 19, pp. 1385-1394, July. 2001

[5] Q. Zhao, P. C. Cosman, and L. B. Milstein, "Optimal allocation of bandwidth for source coding, channel coding and spreading in cdma systems," IEEE Transactions on Communications, vol. 52, pp. 1797-1808, Nov. 2004.

[6] T. L. Martin and D. P. Siewiorek, "Non-ideal battery properties and low power operation in wearable computing," 3rd International Symposium on Wearable Computers, vol. 18-19, pp. 101-106, Oct. 1999.

[7] T. Fowler, N. S. Cheng, K. Burger, A. Samelis, E. Enobakhare, and S. Rohlfing, "Efficiency improvement techniques at low power levels for linear CDMA and WCDMA power amplifiers," IEEE Radio Frequency Integrated Circuits Symposium, 2002.

[8] Q. Zhao, P. C. Cosman, and L. B. Milstein, "Optimal PSK modulation alphabet size and source code rate for a wireless communication system with bandwidth and battery constraints," IEEE Topical Workshop on Power Amplifiers for Wireless Communications, Sept. 2003.

[9] Q. Zhao, Bandwidth Allocation and Tradeoffs of Source Coding, Channel Coding, and Spreading for CDMA Systems. Ph.D Thesis, UCSD, June 2004.

[10] G. D. Forney and G. Ungerboeck, "Modulation and coding for linear Gaussian channels," IEEE Trans. Information Theory, vol. 44, pp. 2384 2415, Oct. 1998

[11] G. Ungerboeck, "Channel coding with multilevel/phase signals," IEEE Trans. Information Theory, vol. 28, pp. 55-67, Jan. 1982.

[12] F. Q. Wang and D. J. Costello, "Probabilistic construction of large constraint length trellis codes for sequential decoding," IEEE Trans. Communications, vol. 43, pp. 2439-2448, Sept. 1995.

[13] L. L. Yang and L. Hanzo, "A recursive algorithm for the error probability evaluation of M-QAM," IEEE Communications Letters, vol. 4, pp. 304 306, Oct. 2000.

[14] J. Lu, K. B. Letaief, J. C.-I. Chuang, and M. L. Liou, "M-PSK and MQAM BER computation using signal-space concepts," IEEE Trans. Communications, vol. 47, pp. 181-184, Feb. 1999. 